\title{
Development and application of an informatization upgrade device for old equipment in ceramic sanitary ware industry
}

\author{
Shi Shanhai ${ }^{1,2}$, Lv Jianqun ${ }^{1,2}$, Xu Yongqian ${ }^{1,2}$, Xie Xiangyong ${ }^{1,2}$ and Zhong Haoxian ${ }^{1,2}$ \\ ${ }^{1}$ Institute of Intelligent Manufacturing, Guangdong Academy of Sciences, Guangzhou, Guangdong, 510000, China \\ ${ }^{2}$ Guangdong Key Laboratory of Modern Control Technology, Guangzhou, Guangdong, 510000, China
}

\begin{abstract}
A device is proposed, which can be used to upgrade the informatization process of old equipment and information isolated island equipment of ceramic enterprises. It has the characteristics of standard, high efficiency, low cost and low risk. The universal fast interface does not interfere with the control system of the device itself when connected to the device. It can obtain data that the target device system has but is not open or the system does not have. Then, the device use the standard industrial communication protocol to transmit the data to the manufacturing execution system in accordance with the agreed data format. This method lay a good foundation for the informatization upgrade and optimization of the data. Furthermore it can be used to optimize and control production. Finally, in enterprise applications, it shows that the device has certain economic and social benefits.
\end{abstract}

\section{Preface}

The transformation and upgrading of the manufacturing industry is the current development trend of the manufacturing industry, and informatization is a direction for the transformation and upgrading of manufacturing enterprises, which has a profound and long-term impact on manufacturing enterprise[1-3]. However, informatization requires accurate, real-time, and effective data. The current problem of manufacturing companies is that most of their old equipment have no access to MES. It caused manufacturing companies to settle for the current status and continue to be information isolated island, and manufacturing management continues to be extensive and blind, without precise informatization. On the other hand, they could customize the software system, customize the communication interface or replace the equipment at high cost, etc. to bridge the connection between the equipment layer and the information layer. No matter which way the enterprise adopts, it is a serious obstacle to the transformation and upgrading of the enterprise. It not only brings problems to the enterprise's manufacturing management, but the additional labor cost, time cost, and asset cost will increase the operating burden of the enterprise.

In the technical services for domestic small and medium ceramic enterprises, most of the equipment in the enterprises is generally old relay equipment or conservative information island equipment. The equipment itself cannot be open to transfer data to MES or the designer does not consider opening data because of security. So that manufacturing companies cannot directly and conveniently obtain data they needed from the equipment, which cuts off the bridge from equipment layer to information layer. Therefore, a cost-effective solution is urgently needed.

\section{Frame and hardware composition}

As the figure 1 shows, the device can operate and process the MES downlink data, automatically operate the production information, such as the start and completion data. Then it sends messages to the underlying equipment to control production. The device execute data collection and data processing of the modified equipment. The device input production data, status data, etc. into the algorithm models in real time to assist production control. Then it upload the data to the MES through the cache interface for subsequent analysis by the MES. The device has the function of alarm operation for abnormal data, and can quickly respond to production abnormalities. The device adopts a near-device deployment method to ensure the stability and reliability of the hardware link. When the upper-layer network fails, the device itself caches production control data. During the network recovery period, the bottom-layer equipment can still be scheduled to continue production, which enhances the stability of production. 


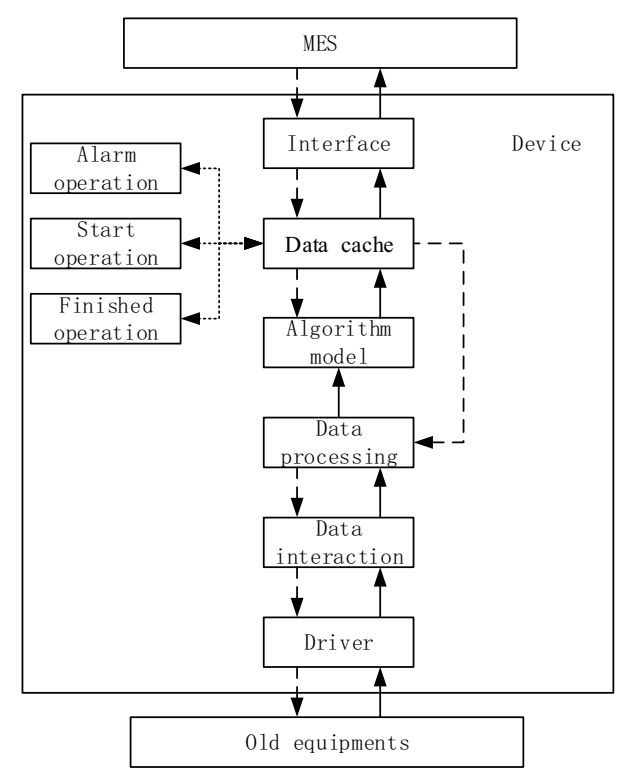

Fig 1. Frame of device.

As mentioned above, this device is used for data acquisition and production control of equipment-level informatization upgrade and transformation, and has a certain versatility. Figure 2 shows the hardware composition. The hardware includes standard power modules, digital input and output modules, analog input and output modules, extended communication modules, standard fast interface modules and so on. The integration and cooperation of the major modules can basically complete the acquisition function of all data needed of the old equipment. All the processed data are used by the information manufacturing execution system.

The standard power module provide energy sources for the device, is composed of circuit breakers, fuses, switching power supplies, and power filters, et al. Some special transformation and upgrading occasions require the addition of lightning protection devices.

The digital input and output modules are mainly composed of a relay modules and a photoelectric coupling modules. They can be connected to the collector to realize the input and output functions of weak current switch signals, strong electricity switch signals, and low voltage pulses, et al.

The analog input and output modules are mainly composed of online distribution modules. The modules have online interchangeability of analog signals such as thermocouple signals, $\mathrm{mA}$ current signals, and weak voltage signals that are common in industrial environments. Some special equipment upgrade need to increase the protection isolation module. Uniformly use isolated electrical wiring to ensure the reliability of the analog transmission link.

The expansion communication modules are mainly composed of the communication hardware linked to the collector, which solves some non-standard protocol communication problems.

The standard quick interface module is the electrical connection interface between the device and the equipment to be upgraded. The wiring hardware are quick

\begin{tabular}{|c|c|c|c|}
\hline \multicolumn{4}{|c|}{$\begin{array}{c}\text { Standard industrial communication } \\
\text { interface }\end{array}$} \\
\hline \multicolumn{4}{|c|}{ Collector } \\
\hline $\begin{array}{c}\text { Power } \\
\text { module }\end{array}$ & $\begin{array}{c}\text { DI D0 } \\
\text { Module }\end{array}$ & $\begin{array}{c}\text { AI A0 } \\
\text { module }\end{array}$ & $\begin{array}{c}\text { Communication } \\
\text { module }\end{array}$ \\
\hline \multicolumn{3}{|c|}{ Standard fast interface module } \\
\hline
\end{tabular}

Fig 2. Hardware composition.

interfaces, and the transformation efficiency is high. The standard interface is functionally partitioned, with strong electromagnetic interference signal data sources, which need to be connected to the designated fast interface area, and ensure isolation on the link from the data sources that are susceptible to electromagnetic interference to ensure the reliability and stability of the data source obtained by the device.

The device is equipped with a standard grounding copper bar, which shares the same ground with the equipment to be upgraded, which minimizes the interference in the process of data source acquisition.

\section{Control software development}

The control software is developed for the function of the device, which is mainly composed of programs and algorithms such as comprehensive control, calculation model, communication driver, interface driver, data processing, and data cache. Due to space limitations, the following will make a brief introduction of the development of some of the critical industry production control related algorithms in the control software and the interactive interface with MES[4-7].

\subsection{Industry production control and algorithms development}

The production control objects of the ceramic sanitary ware industry mainly include automation equipment and operating staff. The equipment mainly includes raw material production equipment, molding equipment, glaze spraying line, polishing line, kiln, etc. Taking the highpressure grouting machine as an example, a process monitoring model and a high-pressure grouting machine capacity calculation model is established for equipment control needs. The formula (1) is the calculation model of the high pressure grouting machine productivity.

$$
\left[P r_{1}, P r_{2}, \cdots, P r_{n}\right]=\alpha\left[M c_{1}, M c_{2}, \cdots, M c_{n}\right]^{T} \cdot\left[P m_{1}, P m_{2}, \cdots, P m_{n}\right]
$$


In formula (1), $\operatorname{Pr}$ represents the actual capacity utilization rate of the specified product in the work order, $M c$ is the reciprocal of the number of molds of the corresponding model in the work order, and $\alpha$ is the reciprocal of the mold opening and closing count of the work order $(M c, \alpha$ are passed from the high pressure grouting machine through the device obtained directly in the process parameter area), $\mathrm{Pm}$ is the quantity reported in the full inspection process for the corresponding model in the work order (downstream from the MES to the buffer area). $n$ is the serial number of the specified model mold. After the device calculates and obtains the actual capacity utilization rate of the specified model of the work order, the data is returned to the MES to assist the MES to realize the capacity statistics and production adjustment scheduling functions.

The high-pressure grouting machine has a complicated process and a mixed production of multiple molds. The process parameter configuration method is generally set

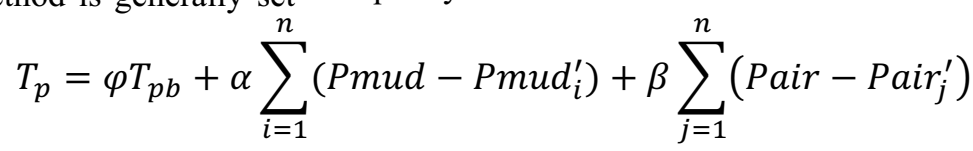

In the formula (2), $T_{p}$ represents the pressure time, $\varphi$ is the correction coefficient associated with mud flow rate, particle size and other related parameters, $T_{p b}$ is the reference pressure time, Pmud is the mud pressure setting parameter, and $P_{m u d}^{\prime}$ is the transient mud pressure, Pair is the compressed air pressure setting parameter, and Pair $_{j}^{\prime}$ is the transient compressed air pressure, $\alpha$ and $\beta$ are correction coefficients, and $n$ is the number of periodic acquisitions.

\subsection{Interactive interface development with MES}

The device interacts with the MES system by developing standard interface communication module. The acquisition communication module is connected with the above modules to complete the device data processing function. This module has a standard industrial communication interface, including but not limited to OPC, Modbus TCP, S7 and other standard industrial protocols. In order to guarantee the reliable data interaction with the upper manufacturing execution system, the function of the collector to the data cache can be added. Figure 3 is the introduction of the interactive mode related to work order. The solid wireframe is the

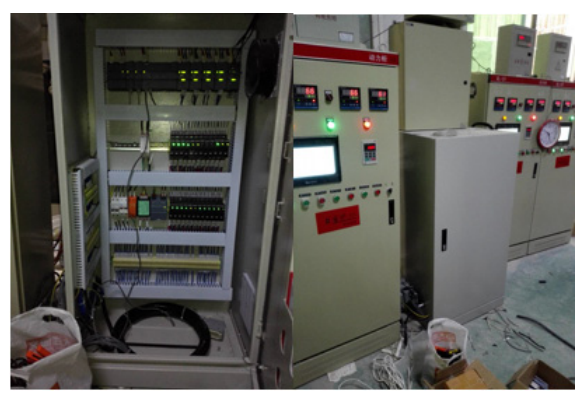

Fig 4. Installation.

The application case is in a ceramics factory in Chaoan by employees based on experience, which has certain risks. If only through information technology to optimize difficult for the MES running in the management level to ensure the stability of production quality. Therefore, it is necessary to establish a process monitoring model, and optimize key parameters through the learning and analysis of MES production big data module to make the process more reliable and stable, and at the same time create conditions for the intelligent upgrade of old equipment. Formula (2) is the calculation model of pressurizing time. After accumulating a large amount of production experience data related to pressurization time, MES obtains the most solvable model correction parameters through a self-learning algorithm. At the same time, the correction coefficient is sent to the device to realize the real-time adjustment and control of the pressurization time, and improve the stability and efficiency of the production quality.

device work order state operation, and the dotted wireframe is the MES work order state and operation. The delivery, scheduling and feedback of the work order are realized through the interactive information of the interface.

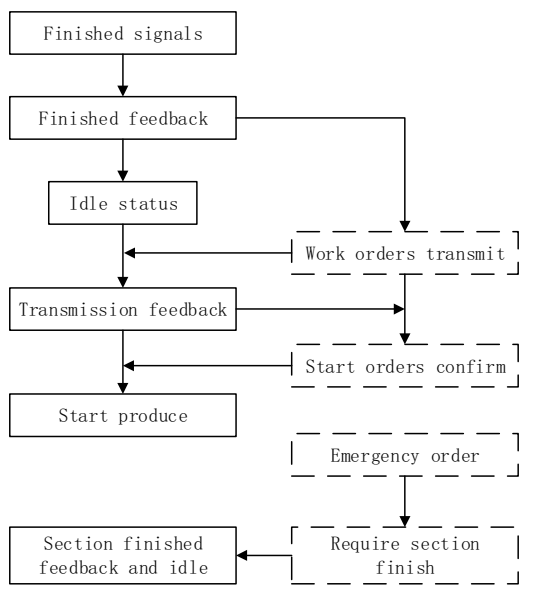

Fig 3. Diagram of an interface information flow.

\section{Application case}

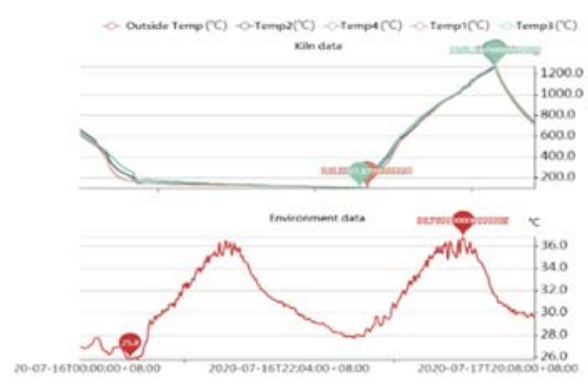

Fig 5. Control curve of kiln.

Districta, Chaozhou, Guangdong Province. The factory production, due to time delay and other reasons, it is 
needs information transformation and upgrading, but all of its equipment are old and has no function of directly connecting with MES. If the function is added to the equipment' control systems, the project cost will exceed half of the total equipment price, which will increase the burden on the enterprise. After discussion and research, the device is installed, as shown in the figure 4. Because of the modular deployment, the entire hardware implementation cycle is only 6 working days, and the implementation efficiency is greatly improved. 4 shuttle kilns and 4 high-pressure grouting machines have been transformed by this device, all of which are equipment needed to be transformed of the ceramics factory. At present, they have been running stably. The designed optimization algorithm has also guided the production of the enterprise to some extent. All equipment data is processed and uploaded to MES. Figure 5 is MES data correlation analysis chart of Furnace NO. 4.

\section{End}

By means of bypass and isolation and so on, the device can obtain the information without affecting the normal operation of the device itself. The control model of high pressure grouting machine, shuttle kiln and other equipment is established, which can analyze and optimize the equipment control process in real time. The device collects part of the equipment load information, more intuitive display the status of equipment and production. Practice has proved that enterprises can use this equipment, with less cost input, promote enterprise information transformation and upgrading, greatly reduce the burden of enterprises.

\section{Acknowledgments}

This work is supported in part by the frontier and key technological innovation special foundation of Guangdong province (Major science and technology project), China, Grant 2016B090912006, the GDAS'
Project of Science and Technology Development, China, Grant 2017GDASCX-0115 and the GDAS' Project of Science and Technology Development, China, Grant 2018 GDASCX-0115.

\section{References}

1. Zhou J.J., Yao X.F., Liu M., Zhang J.M., Tao T. (2017) State of Art review on new emerging intelligent manufacturing paradigms. J. Computer Integrated Manufacturing Systems, 23:624-639

2. Hyoung Seok Kang, Ju Yeon Lee.et al. (2016) Smart manufacturing: past research, present findings, and future directions. J. International Journal of Precision Engineering and Manufacturing Green Technology, 3:111-128

3. Zeng C.Y., Fan Y.L., Wang H.N., Li D.F., Guo C.C. (2020) Green Production And Intelligent Manufacture the Way to Transform And Upgrade of Ceramic Industry. J. Ceramics 6,45-47

4. Jose Salcedo Hernandez,Raul Rivas-Perez,Javier J. (2018)Sotomayor Moriano. Design of a Generalized Predictive Controller for Temperature Control in a Cement Rotary Kiln .J. IEEE Latin America Transactions, 16:1015-1021

5. Hou Y.K., Li Y., Zhang J., Jiang S.S. (2020) Algorithm for transient throughput analysis of assembly systems with automatic and operator based workstations. J. Computer Integrated Manufacturing Systems, 26:1162-1171

6. Liu J.K., Hao S.H., Wang S.H., Fu H.Y. (2019) Data driven technical framework of real-time monitoring and control optimization for $\mathrm{CNC}$ machining production line. J. Computer Integrated Manufacturing Systems, 25:1875-1884

7. Xiong R, Li X.G. (2020) Battery Internal Temperature Estimation Method through Double Extended Kalman Filtering Algorithm. J. Journal of Mechanical Engineering, 56:146-151 